\title{
Optimisation and assessment of theoretical impurity line power coefficients relevant to ITER and DEMO
}

\author{
S. S. Henderson ${ }^{1}$, M. Bluteau ${ }^{1}$, A. Foster ${ }^{2}$, A. Giunta ${ }^{3}$, \\ M. G. O’Mullane ${ }^{1}$, T. Pütterich ${ }^{4}$, H. P. Summers ${ }^{1}$ \\ ${ }^{1}$ Department of Physics SUPA, University of Strathclyde, Glasgow, G4 0NG, UK \\ ${ }^{2}$ Smithsonian Astrophysical Observatory, Cambridge, MA 02144, USA \\ ${ }^{3}$ RAL Space, Science and Technology Facilities Council, Rutherford Appleton \\ Laboratory, OX11 0QX, UK \\ ${ }^{4}$ Max-Planck-Institut für Plasmaphysik, D-85748 Garching, Germany \\ E-mail: stuart.s.henderson@strath.ac.uk
}

\begin{abstract}
Total radiated line power coefficients for ions of medium to heavy weight elements, called $\mathcal{P} \mathcal{L} \mathcal{T}$ coefficients in the Atomic Data and Analysis Structure (ADAS), have been improved by algorithmically optimising the selection of configuration sets that underpin the calculation to include the most important radiating transitions driven by both the ground and metastable configurations and to establish and limit the error of truncation. The optimised calculations typically differ from Pütterich by $20-30 \%$ with truncation error $\lesssim 5 \%$. Further appraisal of error due to atomic level bundling, atomic structure and collision strength calculation methods has been carried out. It is shown that bundling to configurations is accurate to $\lesssim 10 \%$ for all ions except those with closed-shell ground configurations which give errors up to a factor $2-3$. For near neutral, closed-shell ions, plane-wave Born collision strength calculations, which omit spin-change, give substantial error in comparison with distorted-wave calculations of $\mathcal{P} \mathcal{L} \mathcal{T}$. For highly charged ions, spin-system breakdown reduces the error in the $\mathcal{P} \mathcal{L} \mathcal{T}$ markedly, typically $\lesssim 10 \%$. The error introduced by the atomic structure codes used here, autostructure and the cowan code, is probably limited to $\lesssim 30 \%$.
\end{abstract}

\section{Introduction}

Significant effort is currently being directed towards exploring different conceptual DEMO tokamak designs using system codes, such as process and sycomore $[1,2,3]$. One branch of this research concerns the role that impurities play in the core plasma power balance and the divertor protection. In this regard, the steady-state heat loads expected in current baseline designs for ITER are well above the tolerable limit for metallic divertors [4,5]. To reduce these heat loads, radiative cooling by seeding of noble gases (and nitrogen gas) is under consideration for DEMO and ITER [6,7]. Accurate theoretical impurity cooling factors are required to explore the balance between the negative and positive effects of the impurity radiation in the different zones of the plasma. The baseline design scenarios are sensitive to the cooling factors [8], that is 
the combination of the ionisation balance with the total radiated line power, $\mathcal{P} \mathcal{L} \mathcal{T}$, and the Bremsstrahlung and recombination power. The focus here is only on the error of the $\mathcal{P} \mathcal{L} \mathcal{T}$ coefficients. Other coefficients required to establish the ionisation balance are collectively called the collisional-radiative coefficients in the Atomic Data and Analysis Structure (ADAS) [9].

The $\mathcal{P} \mathcal{L} \mathcal{T}$ coefficient is that part of the radiative power driven by excitation (by electron collisions) from the ground and/or metastable states of the ion. For each ion, it requires transition energies, associated radiative transition probabilities, and electron-impact excitation collision strengths (Maxwell averaged) which span an appropriate set of electron configurations. The handling of these configurations can be done at various levels of resolution called bundling representations. Typically these are configuration average $(c a)$, term-coupled $(l s)$ or level resolved intermediate-coupled $(i c)$ representations. $c a$ and $i c$ representations are required for the present study. ADAS have available lower quality, density-independent $\mathcal{P} \mathcal{L} \mathcal{T}$ coefficients for a wide range of elements up to Xe and higher quality density-dependent collision-radiative $\mathcal{P} \mathcal{L} \mathcal{T}$ coefficients which follow the generalised collisional-radiative (GCR) development currently available for elements up to $\mathrm{Ne}[10]$.

A set of consistent ionisation, recombination and $\mathcal{P} \mathcal{L} \mathcal{T}$ coefficients for elements up to $\mathrm{W}$ will be added to the ADAS in the near future from Pütterich which follow the calculation methodology of previous studies [11, 12]. For this data set, the $\mathcal{P L T}$ coefficients are generated using the cowan code [13] (CW) with a plane-wave Born (PWB) approximation to calculate the electronic structure and electron-impact excitation collision strengths. In the general case, the electron configuration sets underpinning each iso-electronic sequence calculation are those from the $\mathrm{W}$ configuration sets described by Pütterich et al [11]. For this work, these $\mathcal{P} \mathcal{L} \mathcal{T}$ coefficients have been improved for each specific ion by selecting the configuration sets with a novel, computerised optimisation process based on previous work by Foster [14] ensuring the inclusion of all the significant contributing configurations. Additionally, an assessment is made of the validity of using: a PWB approximation in comparison to a higher quality distorted wave (DW) approximation calculated using the autostructure code [15, 16] $(\mathrm{AS})$; a $\mathcal{P} \mathcal{L} \mathcal{T}$ top-up estimate derived from the comparison of a $c a$ calculation with a large and small electron configuration set; and a model which neglects both spinchanging and ion-impact excitation collision strengths.

In this paper, section 2 describes the algorithmic method for optimising the electron configuration sets of each ion. The optimised $\mathcal{P} \mathcal{L} \mathcal{T}$ coefficients for a selection of fusion (and astrophysical) relevant elements (Ar, Fe, Kr, Xe, and W) are presented in section 3, followed by an assessment of the error expected for the various approximations used in the calculation. Although experimental measurements of impurity cooling factors are possible [17], the contributions to the cooling factor from the ionisation balance and $\mathcal{P} \mathcal{L} \mathcal{T}$ coefficients are difficult to separate. Instead, theoretical $\mathcal{P} \mathcal{L} \mathcal{T}$ coefficients for $W$ from Pütterich et al [12] and for two Kr ions from Fournier et al [18] are compared. Data archiving details and concluding remarks are given in section 4 and 5 , respectively. 


\section{Configuration selection optimisation}

High precision electron collision calculations for spectral diagnostics may overlook important configurations (for total power) [19]. In this work, the selection of configurations is automated by optimising a set of rules that define excited configurations to be built on an initial (ground/metastable) configuration. For example, the rules dictate the allowed change in the $n$ and $l$ quantum numbers when promoting electrons from valence and closed shells. A full specification of each rule is given in table 1 and discussed in detail by Foster [14].

The optimisation of these rules for one initial configuration (i.e. the ground configuration) is based on producing the maximum value of a single $\mathcal{P} \mathcal{L} \mathcal{T}$ coefficient defined as

$$
\mathcal{P} \mathcal{L} \mathcal{T}=\frac{N_{1}}{N_{\text {total }}} \sum_{i, j} \Delta E_{i, j} A_{j \rightarrow i} \mathcal{F}_{j, 1}^{\text {exc }}\left[\mathrm{Wm}^{3}\right] .
$$

$i$ and $j$ represent the lower and upper levels spanning the atomic energy levels defined in the electron configuration sets, $\Delta E_{i, j}$ is the $j \rightarrow i$ transition energy, $A_{j \rightarrow i}$ is the spontaneous emission coefficient, $\mathcal{F}_{j, 1}^{e x c}$ is the component of the population of the $j$ th level associated with the ground level divided by the electron density, and $N_{1}$ and $N_{\text {total }}$ represent the ground and total population of the ion.

The code, called adas808 in ADAS, works as follows. Firstly, as input, the code requires a reference electron temperature $T_{e}$ and density $n_{e}$, the element and ionisation stage, and the driving configuration. Here, $T_{e}$ and $n_{e}$ are set equal to the ionisation potential IP of the ion and a density of $10^{19} \mathrm{~m}^{-3}$ (typical of fusion plasmas), respectively. Although the temperature of peak ion abundance is lower than $I P$, optimising at $T_{e}=I P$ ensures that the $\mathcal{P} \mathcal{L} \mathcal{T}$ coefficient is in the plateau region rather than in the

Table 1. Promotion rules used to define configuration sets

\begin{tabular}{|l|l|l|l|}
\hline Rule & Description & Rule & Description \\
\hline$\# 1$ & Number of valence shells & $\# 14$ & Promote closed shells (y/n) \\
$\# 2$ & Max $\Delta n$ (1st valence shell) & $\# 15$ & Max $\Delta n$ promotion (closed shell) \\
$\# 3$ & Min $\Delta n($ st valence shell) & $\# 16$ & Min $\Delta n$ promotion (closed shell) \\
$\# 4$ & Max $\Delta l(1$ st valence shell) & $\# 17$ & Max $\Delta l$ promotion (closed shell) \\
$\# 5$ & Min $\Delta l($ st valence shell) & $\# 18$ & Min $\Delta l$ promotion (closed shell) \\
$\# 6$ & Max $\Delta n($ nd valence shell) & $\# 19$ & Add configs. of same complex $(\mathrm{y} / \mathrm{n})$ \\
$\# 7$ & Min $\Delta n($ nd valence shell) & $\# 20$ & Shift valence electron to \\
$\# 8$ & Max $\Delta l(2$ nd valence shell) & & unfilled 4 as extra ground \\
$\# 9$ & Min $\Delta l$ (2nd valence shell) & $\# 21$ & Add all $n l$ configurations of \\
$\# 10$ & Max $n$ (closed shell) & & outer valence shell (y/n) \\
$\# 11$ & Min $n$ (closed shell) & $\# 22$ & If \#21 add opposite or both \\
$\# 12$ & Max $l$ (closed shell) & & parities to valence shell \\
$\# 13$ & Min $l$ (closed shell) & $\# 23$ & Cowan specific rules \\
\hline
\end{tabular}


sharply increasing region. Next, each rule is initialised to zero except for rule \#1 and rules \#10 to \#13. The former is set to unity, while the code loops around different values for the latter gradually opening each inner shell. Within each of these loops, the code progresses iteratively by sequentially performing a set of rule changes (defined in table 2). If the rule change produces a new valid set of configurations $\ddagger$, then the $\mathcal{P} \mathcal{L} \mathcal{T}$ coefficient (defined in equation 1) is determined. The atomic energy levels, spontaneous emission coefficients and collision strengths are calculated using $\mathrm{CW}$ in $c a$ mode. After all rule changes have been attempted, the change producing the largest $\mathcal{P} \mathcal{L} \mathcal{T}$ coefficient is chosen as the reference case if it remains within a set of pre-defined level count, configuration count, and power threshold limits. For this analysis, a small configuration set is defined with a level count limit of 1000, configuration count limit of 30, and a power threshold of $0.001 \%$. Large configuration sets, which only adhere to the power threshold limit, are used to estimate the top-up corrections and are discussed in more detail in section 3 . The optimisation process then continues using the new rule set as the initial conditions for the next set of changes.

The optimisation of the C-like $\mathrm{Ar}^{12+}$ and Ca-like $\mathrm{Kr}^{16+}$ configuration sets is shown in figure 1. The level and configuration count limits were relaxed allowing the code to run until either the change in the power was below $0.001 \%$ or until the rule changes produced no further valid configuration sets. A convergence in the $\mathcal{P} \mathcal{L} \mathcal{T}$ coefficient is found for both ions despite the linear increase in levels at the end of each iteration. For less complex structure calculations, such as the C-like ground configuration, this convergence occurs within a total level count set by computational restraints. More $\ddagger$ If $\mathrm{CW}$ fails to converge, then the given set of electron configurations are deemed invalid.

Table 2. Rule change sequence during each iteration of the optimisation code adas 808 . \# numbers correspond to the defined rules given in table 1 .

\begin{tabular}{|c|c|c|c|c|c|c|}
\hline \multicolumn{4}{|c|}{ Iteration 1} & \multicolumn{3}{|c|}{ Iteration $>1$} \\
\hline 1: $\quad \# 4=-1$ & $6: \quad \# 6=1$ & 10: & $\# 17=-1$ & $1: \quad \# 2=\# 2+1$ & & $\# 22=1$ \\
\hline$\# 5=-1$ & $\# 7=1$ & & $\# 18=-1$ & $2: \quad \# 3=\# 3-1$ & 15: & $\# 21=1$ \\
\hline 2: $\quad \# 2=1$ & $\# 8=-1$ & 11: & $\# 17=1$ & $3: \quad \# 4=\# 4+1$ & & $\# 22=0$ \\
\hline$\# 3=1$ & $\# 9=-1$ & & $\# 18=1$ & 4: $\quad \# 5=\# 5-1$ & 16: & $\# 20=1$ \\
\hline$\# 4=-1$ & 7: $\quad \# 8=1$ & 12: & $\# 15=1$ & $5: \quad \# 6=\# 6+1$ & 17: & $\# 2=\# 2+1$ \\
\hline$\# 5=-1$ & $\# 9=1$ & & $\# 16=1$ & $6: \quad \# 7=\# 7-1$ & & $\# 4=\# 4+1$ \\
\hline 3: $\quad \# 4=1$ & 8: $\quad \# 6=1$ & & $\# 17=1$ & $7: \quad \# 8=\# 8+1$ & 18: & $\# 6=\# 6+1$ \\
\hline$\# 5=1$ & $\# 7=1$ & & $\# 18=1$ & 8: $\quad \# 9=\# 9-1$ & & $\# 8=\# 8+1$ \\
\hline 4: $\quad \# 2=1$ & $\# 8=1$ & 13: & $\# 21=1$ & $9: \quad \# 15=\# 15+1$ & 19: & $\# 15=\# 15+1$ \\
\hline$\# 3=1$ & $\# 9=1$ & & $\# 22=1$ & $10: \quad \# 16=\# 16-1$ & & $\# 17=\# 17+1$ \\
\hline$\# 4=1$ & 9: $\quad \# 17=-1$ & 14: & $\# 21=1$ & 11: $\quad \# 17=\# 17+1$ & & \\
\hline$\# 5=1$ & $\# 18=-1$ & & $\# 22=0$ & 12: $\quad \# 18=\# 18-1$ & & \\
\hline 5: $\quad \# 8=-1$ & 10: $\quad \# 15=1$ & & & 13: $\quad \# 19=1$ & & \\
\hline$\# 9=-1$ & $\# 16=1$ & & & 14: $\quad \# 21=1$ & & \\
\hline
\end{tabular}



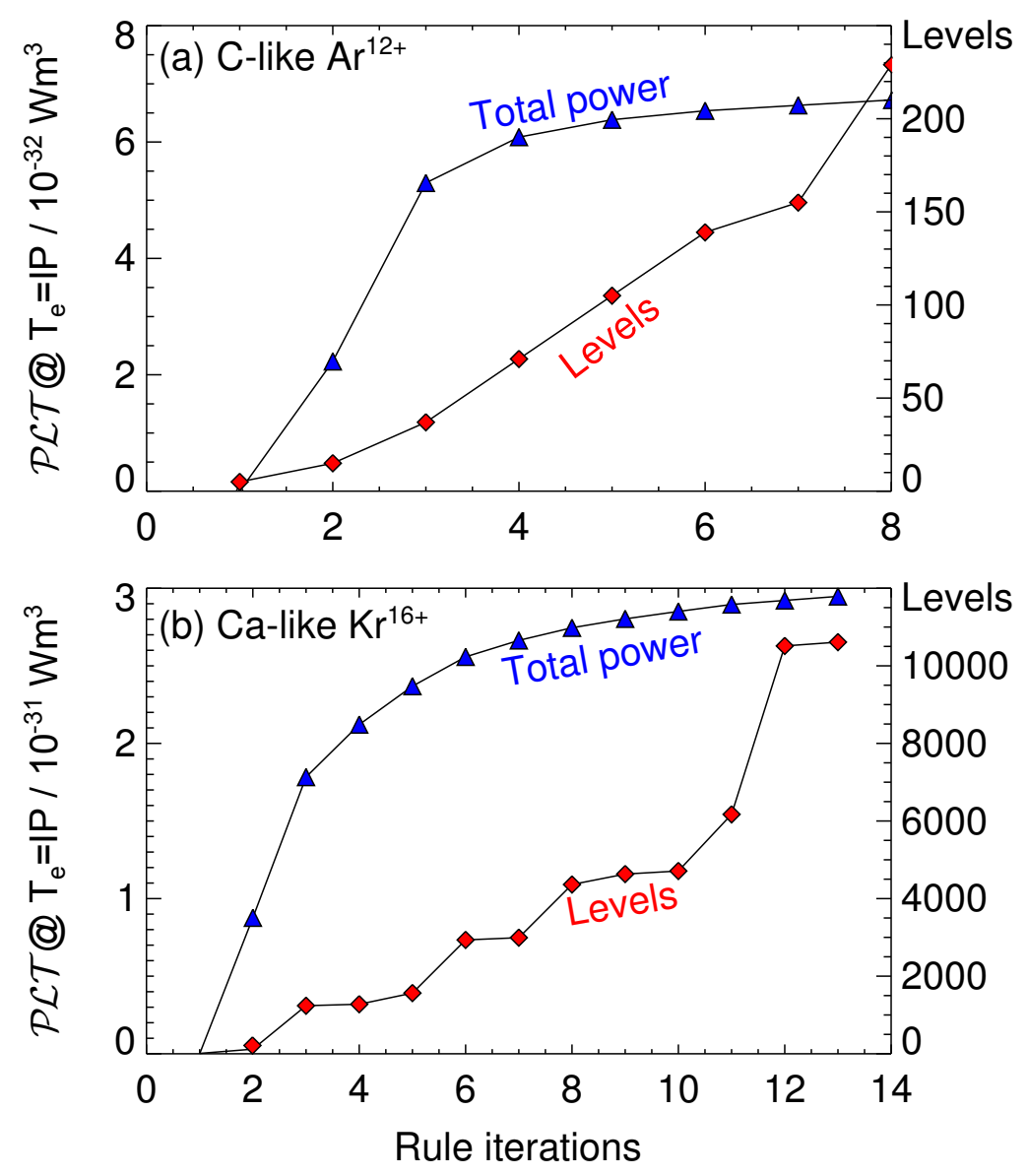

Figure 1. Optimisation of the (a) C-like $\mathrm{Ar}^{12+} 2 \mathrm{~s}^{2} 2 \mathrm{p}^{2}$ ground configuration and the (b) Ca-like $\mathrm{Kr}^{16+} 3 \mathrm{~s}^{2} 3 \mathrm{p}^{6} 3 \mathrm{~d}^{2}$ ground configuration. The blue triangles show the radiated line power selected at the end of each iteration and the red diamonds indicate the associated number of levels. The radiated power is calculated using $T_{e}=I P$ and $n_{e}=10^{19} \mathrm{~m}^{-3}$.

complex ions with an open 3d-, 4d-, or 4f- shell, such as the Ca-like case, typically require a far higher number of levels before convergence in the $\mathcal{P} \mathcal{L} \mathcal{T}$ coefficient is achieved.

\subsection{Metastable optimisation}

Metastable configurations with populations similar to that of the ground configuration often exist in complex ions in low-density plasma. The ratio of the metastable and ground configuration populations are shown in figure 2 for ions with a Be-like, Mg-like, and V-like ground configuration. Typically, the ratios drop below $\approx 10^{-3}$ when the ion charge $Z>5$. Highly populated metastable configurations can offer new pathways for electron promotion and therefore the optimisation procedure should account for promotions from multiple driving (ground and metastable) configurations. Furthermore, since a highly populated metastable can also act to decrease the $\mathcal{P} \mathcal{L} \mathcal{T}$ coefficient due to the factor $\frac{N_{1}}{N_{\text {total }}}$ in equation 1, all known metastables are included in the final configuration sets even if their contribution to the total power is negligible (or negative). 


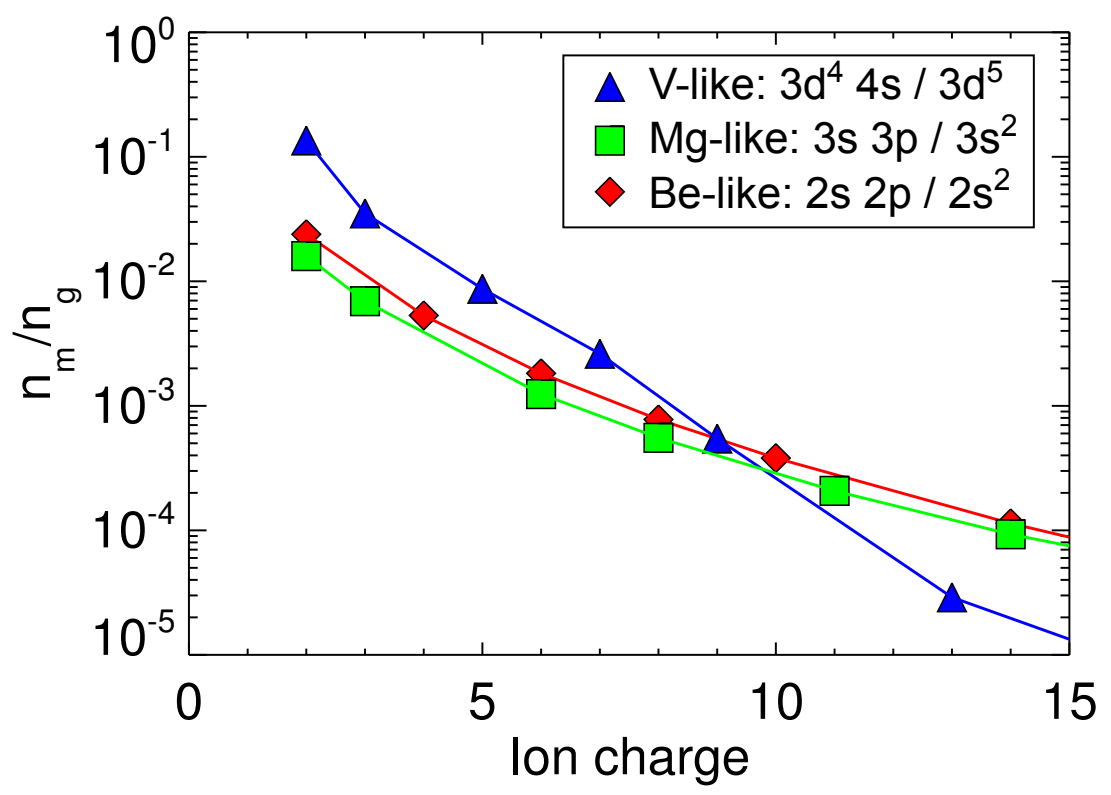

Figure 2. The ratio of the metastable and ground configuration populations for ions in the Be-like (red curve, triangle symbols), Mg-like (green curve, square symbols), and V-like (blue curve, diamond symbols) iso-electronic sequences calculated using the ADAS collisional-radiative model.

The optimisation procedure detailed in the previous subsection is automatically carried out for each metastable driving configuration. Adding together all of the configurations derived from the promotion rules associated with each metastable will usually raise the number of levels above the original level limit (even after removing any duplicate configurations). In extreme cases, CW will fail to converge with the large number of configurations included in the calculation. Therefore, two additional optimisation steps are required to reduce the number of configurations. When $\mathrm{CW}$ fails to converge, the optimisation code removes all configurations with quantum number $n>n_{\text {grd }}+2$, where $n_{\text {grd }}$ is the quantum number of the ground shell. If this condition is not met, then the highest quantum number $n$, even parity configurations promoted from the metastables are removed. The parity condition is removed if a convergence is still not reached.

With a valid configuration set, a further reduction is performed to keep the calculation within the limits. First, the ground and metastables are included along with the first excited configuration (of opposite parity) to ensure that the metastable is tied to at least one other configuration. Next, a collisional-radiative population calculation is carried out to determine $\mathcal{F}_{j, 1}^{e x c}$ and then the configuration transition pairs are arranged in descending order in terms of their individual contribution to the total power, $\Delta E_{i, j} A_{j \rightarrow i} \mathcal{F}_{j, 1}^{\text {exc }}$. The configuration pair is included if the calculation remains within the calculation limits. After the addition of a configuration pair, the dominant (de-)populating configurations associated with the upper configuration are included.

Inclusion of the metastables can have a varied effect on the final $\mathcal{P} \mathcal{L} \mathcal{T}$ coefficient. 
Consider the metastables from Ca-like to Ni-like which include the ground configuration $3 \mathrm{~d}^{i}$ and the two metastable configurations $3 \mathrm{~d}^{i-1} 4 \mathrm{~s}$ and $3 \mathrm{~d}^{i-2} 4 \mathrm{~s}^{2}$ where $1 \leq i \leq 10$. For the weakly ionised species, the first metastable is often included in the initial optimisation of the ground configuration because of its ability to populate the $3 \mathrm{~d}^{i-1} 4 \mathrm{p}$ configuration which enhances the strongly radiating $3 \mathrm{~d}^{i-1} 4 \mathrm{p} \rightarrow 3 \mathrm{~d}^{i}$ transition. The secondary metastable optimisation step therefore only causes a minor reduction in the $\mathcal{P} \mathcal{L} \mathcal{T}$ coefficient due to the addition of the $3 \mathrm{~d}^{i-2} 4 \mathrm{~s}^{2}$ configuration. As the ion charge increases the re-normalisation (that is to the total ion population, but in effect to the sum of ground and metastable populations) of the $\mathcal{P} \mathcal{L} \mathcal{T}$ coefficient outweighs the additional line-power and therefore neither the $3 \mathrm{~d}^{i-1} 4 \mathrm{~s}$ or the $3 \mathrm{~d}^{i-2} 4 \mathrm{~s}^{2}$ configuration is included in the initial optimisation. In these cases, the inclusion of the metastables can decrease the $\mathcal{P} \mathcal{L} \mathcal{T}$ coefficient by $20-30 \%$. For highly charged ions, where the population of the metastable configurations listed above are considerably lower than the ground population, the secondary metastable optimisation typically only causes a modest increase in the $\mathcal{P} \mathcal{L} \mathcal{T}$ coefficient of $\leq 5 \%$.

\section{Optimised $\mathcal{P} \mathcal{L} \mathcal{T}$ coefficients}

The optimised $\mathcal{P} \mathcal{L} \mathcal{T}^{\text {tot }}$ coefficients (see equation 2) shown in figure $3 \mathrm{~b}$ have been determined using $n_{e}=10^{19} \mathrm{~m}^{-3}$, the configuration sets discussed in the previous section, and the temperatures of peak ion abundance (shown in figure $3 \mathrm{a}$ ). The optimised $\mathcal{P L T}$ coefficients for the closed shell ions (Ar-like, Kr-like, and Pd-like) have been supplemented with true spin-changing collision strengths calculated using AS with the DW approximation. The following subsections discuss the accuracy of these $\mathcal{P} \mathcal{L} \mathcal{T}$ coefficients based on the various calculation methodologies.

\subsection{Top-up estimate}

Ideally, the additional line power due to excluded levels should be calculated using the projection matrices of GCR theory (Summers et al [10]). Currently, this data is only available in term resolved resolution for species up to neon and therefore a simpler top-up estimate is adopted for the heavier elements calculated as

$$
\mathcal{P} \mathcal{L} \mathcal{T}^{t o t}=\mathcal{P} \mathcal{L} \mathcal{T}^{i c(c s)} \frac{\mathcal{P} \mathcal{L} \mathcal{T}^{c a(c l)}}{\mathcal{P} \mathcal{L} \mathcal{T}^{c a(c s)}}
$$

where the $i c$ and $c a$ superscripts denote the calculation resolution spanning the small $(c s)$ and large $(c l)$ configuration set sizes. The accuracy of the ca calculations remains approximately constant as the configuration set size is increased since the extended configuration set usually includes states of higher- $n$ where configuration bundling is most accurate (as described in the next sub-section).

Figure 4 compares $\mathcal{P} \mathcal{L} \mathcal{T}^{c a(c l)}$ and $\mathcal{P} \mathcal{L} \mathcal{T}^{c a(c s)}$ coefficients for $\mathrm{W}, \mathrm{Kr}$, and Fe. The 

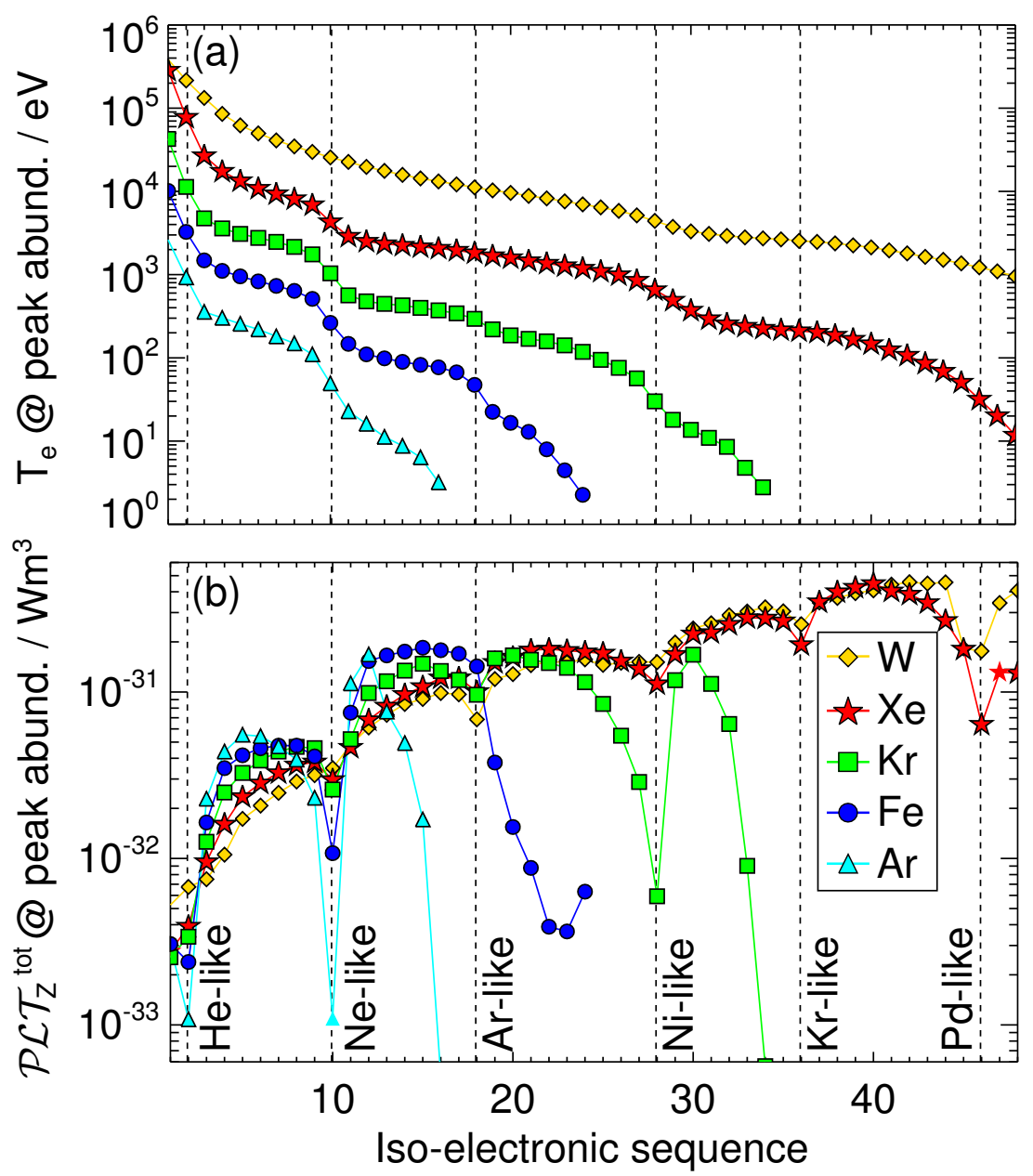

Figure 3. (a) The temperatures of peak abundance for each ion found using the ionisation and recombination coefficients defined by Pütterich [12] and (b) the corresponding $\mathcal{P} \mathcal{L} \mathcal{T}^{t o t}$ coefficients (see text, equation 2) at each temperature given in (a) determined using CW.

differences are defined in terms of a $\Delta$ factor,

$$
\Delta\left(y_{1}, y_{2}\right)=\frac{\max \left(\left\{y_{1}, y_{2}\right\}\right)}{\min \left(\left\{y_{1}, y_{2}\right\}\right)}-1,
$$

where $y_{1}$ and $y_{2}$ denote the two values that are under examination. A representative $\Delta$ error factor for each ion is determined by picking the value of $\Delta$ at the temperatures of peak ion abundance, which are shown in figure $3 \mathrm{a}$. The largest errors are found in the highly charged ions with an open p-shell (with more than two free holes) and with an open d-shell (with more than two electrons and less than two free holes). The latter effect was also reported by Pütterich et al [12].

The highly charged ions with an open p-shell generate a significant proportion of line power from transitions involving the lower n-shells. The small configuration sets have omitted opening closed inner n-shells since CW energy level discrepancies with NIST were markedly worse. This largely explains the differences found for these particular 


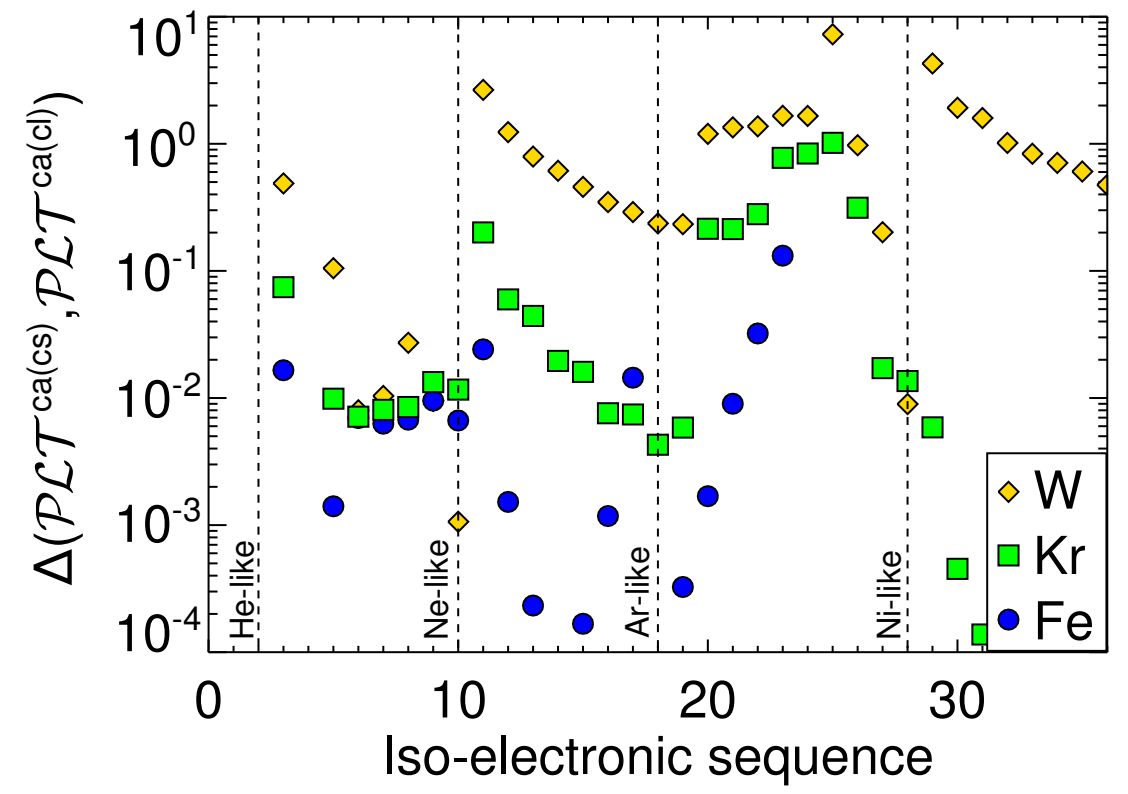

Figure 4. Comparison of $\mathcal{P} \mathcal{L} \mathcal{T}^{c a(c l)}$ and $\mathcal{P} \mathcal{L} \mathcal{T}^{c a(c s)}$ coefficients.

ions. At lower charge, less power is generated through these particular transitions and therefore the difference between the large and small calculations is less. It is also evident that truncation has a smaller impact on low charge ions with an open d-shell. For these ions, the truncation error is most significant when the majority of power emanates from the $4 \mathrm{f} \rightarrow 3 \mathrm{~d}$ transition, since the $4 \mathrm{f}$-shell is excluded in the small configuration sets. However, as the metastability of the ion increases (i.e. as the charge decreases) the distribution of radiated power is skewed in favour of the transitions driven by the metastable configurations which typically involve fewer levels and are therefore generally included within the small configuration sets.

\subsection{Resolution accuracy}

For heavier impurities with complex ground configurations involving an open d- or fshell, the accuracy of the $c a$ calculations must be assessed since $\mathcal{P} \mathcal{L} \mathcal{T}^{i c(c s)}$ coefficients are often not available. For example, the first two excited configurations of the Xe-like $\mathrm{W}^{20+}$ ion, $4 \mathrm{f}^{7} 5 \mathrm{p}$ and $4 \mathrm{~d}^{9} 4 \mathrm{f}^{8}$, generate 1928 and 1878 levels, respectively. The differences between the $\mathcal{P} \mathcal{L} \mathcal{T}^{c a(c s)}$ and $\mathcal{P} \mathcal{L} \mathcal{T}^{i c(c s)}$ coefficients can occur for two reasons. Firstly, in $c a$ resolution the transition energy uses the centroid energy of each configuration, which is an approximation to the weighted mean of levels. For large fine-structure separations this is unsound. Secondly, non-statistical fine-structure populations can occur in ic collisional-radiative modelling which are not accounted for in $c a$ resolution. Consider the $4 \mathrm{~d}^{10}{ }^{1} \mathrm{~S}_{0}$ ground level of $\mathrm{Pd}$-like $\mathrm{W}^{28+}$ and its first excited configuration $4 \mathrm{~d}^{9} 4 \mathrm{f}$ as an example. Of the 20 levels in the open f-shell, only one level has an allowed route (i.e. $\Delta S=0 ; \Delta J=1)$ to the singlet ground level $\left(4 \mathrm{~d}^{9} 4 \mathrm{f}^{1} \mathrm{P}_{1} \rightarrow 4 \mathrm{~d}^{10}{ }^{1} \mathrm{~S}_{0}\right)$. The other fine-structure levels with $\Delta J \geq 2$ are metastable in principle but limited by spin-system 

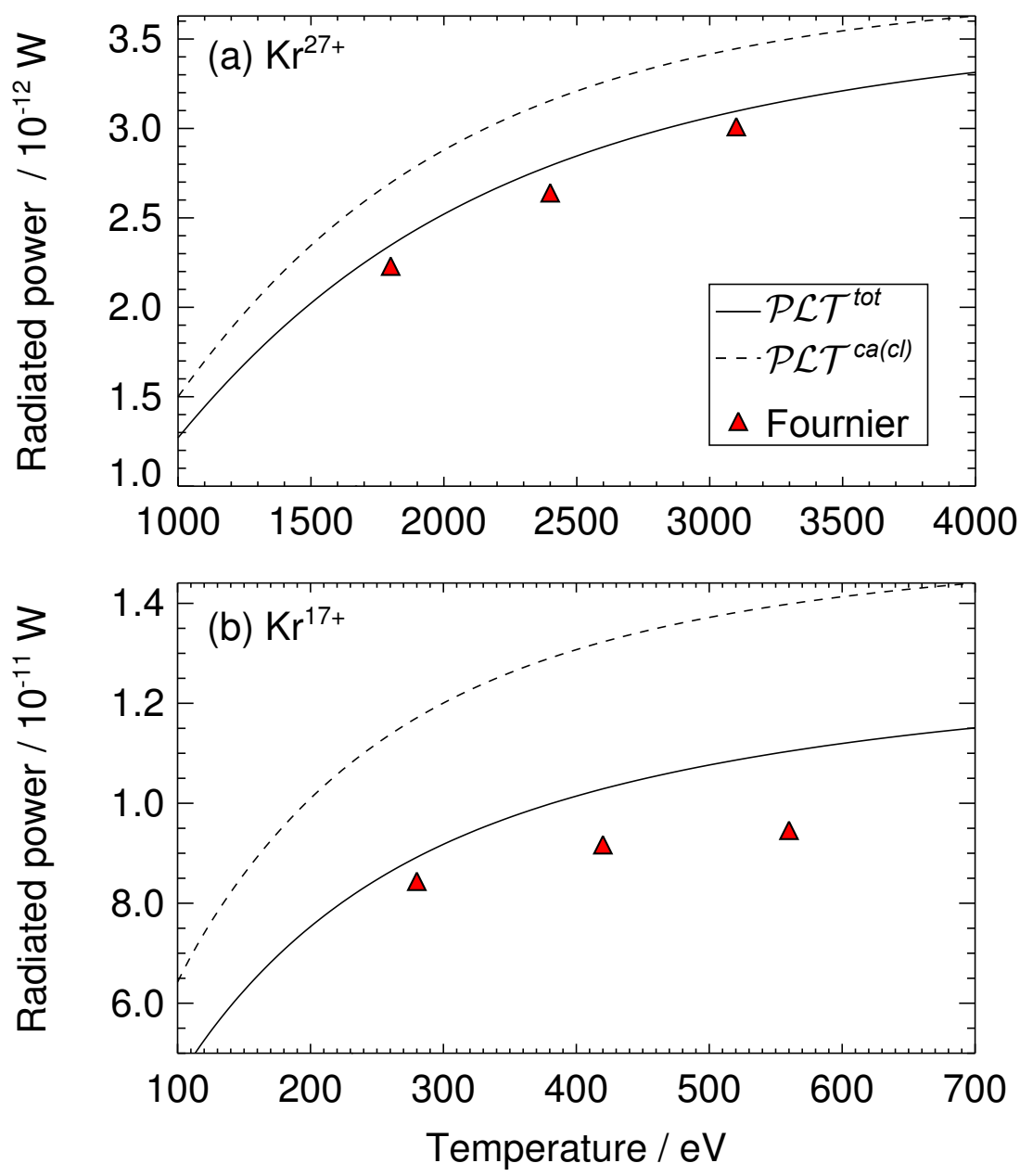

Figure 5. A comparison of the $\mathcal{P} \mathcal{L} \mathcal{T}^{\text {tot }}$ (see text, equation 2) and $\mathcal{P} \mathcal{L} \mathcal{T}^{c a(c l)}$ coefficients at $n_{e}=5 \cdot 10^{19} \mathrm{~m}^{-3}$ for (a) F-like $\mathrm{Kr}^{27+}$ and (b) K-like $\mathrm{Kr}^{17+}$. Equivalent data points from Fournier et al [18] are shown by the red triangles.

breakdown and collisional mixing within the configuration.

The $\mathcal{P} \mathcal{L} \mathcal{T}^{t o t}$ and $\mathcal{P} \mathcal{L} \mathcal{T}^{c a(c l)}$ coefficients for two ions, F-like $\mathrm{Kr}^{27+}$ and $\mathrm{K}$-like $\mathrm{Kr}^{17+}$, are shown in figure $5 \mathrm{a}$ and figure $5 \mathrm{~b}$, respectively. The differences between the two calculations indicate that $c a$ resolution introduces a modest error of $\approx 10 \%$ for the F-like ion and a larger error of $\approx 50 \%$ for the K-like ion. As described above, $c a$ resolution is least accurate for particular closed shell ions. The $Z \pm 1$ ions adjacent to these closed shell ions (i.e. in this case K-like) have similar inaccuracies to the closed shell ions but to a lesser extent. The data points in tables 1 and 2 from Fournier et al [18] are shown by the triangles for comparison. The $\mathcal{P} \mathcal{L} \mathcal{T}^{\text {tot }}$ coefficients from this analysis are $\approx 20 \%$ greater in magnitude for both ions which may either be due to the extra configurations from the optimisation procedure or because of differences in the calculation methodology.

Figure 6 shows the $\mathcal{P} \mathcal{L} \mathcal{T}^{\text {tot }}$ and $\mathcal{P} \mathcal{L} \mathcal{T}^{c a(c l)}$ coefficients for the iso-nuclear sequence of $\mathrm{W}(15 \leq Z \leq 70)$ at the temperatures of peak ion abundance shown in figure 3a. A significant difference is found between the two curves for Pd-like, Kr-like, and 


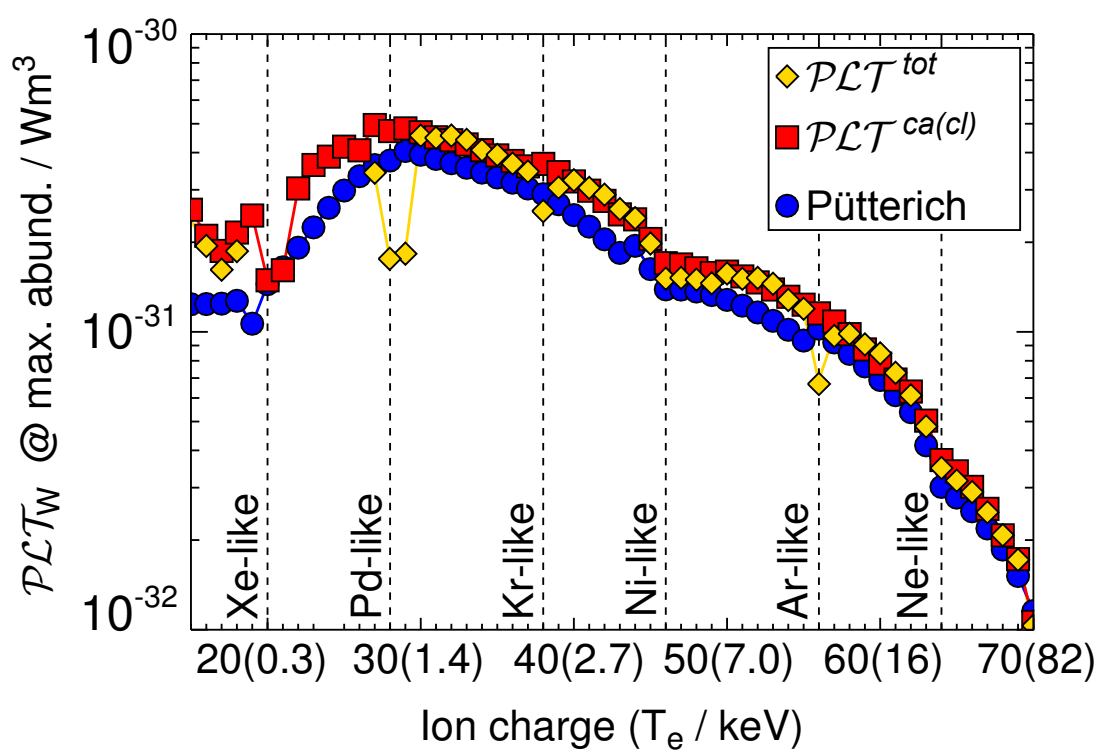

Figure 6. Yellow diamonds and red squares show the $\mathrm{W} \mathcal{P} \mathcal{L} \mathcal{T}^{\text {tot }}$ (see text, equation 2) and $\mathcal{P} \mathcal{L} \mathcal{T}^{c a(c l)}$ coefficients at the temperature of peak abundance, respectively. Equivalent $\mathrm{W} \mathcal{P} \mathcal{L} \mathcal{T}^{c a(c l)}$ coefficients from Pütterich [12] are shown by blue circles.

Ar-like closed shell ions due to a metastability in the 4f-shell, 4d-shell, and 3d-shell respectively. The 3s-shell of the Ne-like ion does not develop any metastability in ca or $i c$ resolution, whereas the 4 s-shell of the Ni-like ion is metastable in both $c a$ and $i c$ resolution. Differences $\lesssim 10 \%$ between the two curves are again indicative of the error introduced by the degree of energy level splitting in comparison to the centroid energy. Also shown for comparison are the equivalent $\mathcal{P} \mathcal{L} \mathcal{T}^{c a}(c l)$ coefficients from Pütterich et al [12]. The inclusion of extra configurations derived from the optimisation method in section 2 typically accounts for an additional $\approx 20-30 \%$ of power for each ion.

\subsection{Collision strength approximations}

When the total population of a configuration of an ion in a plasma is similar for both $i c$ and $c a$ resolutions, then the $\mathcal{P} \mathcal{L} \mathcal{T}$ coefficient accuracy is mainly dependent on the quality of the dipole collision strengths and the associated spontaneous emission coefficients and transition energies. Conversely, if a strong build-up of population occurs in quasimetastable levels within a configuration, as discussed in the previous section, then the inclusion and quality of the non-dipole and forbidden collision strengths matter.

Calculations of the electron-impact excitation collision strengths using CW are in the plane-wave Born PWB approximation - a good high energy approximation but not for spin-changing transitions. Comparisons of the collision strength for dipole transitions calculated using AS with the DW and PWB approximations produce differences of $\lesssim 40 \%$ at low energy $\S$ and converge at high energy. The Maxwell averaged

$\S$ All PWB calculations include a threshold adjustment to give a finite value for electron impact with ions. See Cowan equation 18.160 [13]. 
collision strengths converge in the same manner at high energy, but typically the ionisation balance for heavy elements moves a given iso-electronic sequence ion to higher temperatures where the collision strengths are converging. For the low ionisation stages of heavier elements the threshold region matters more. The preferred DW collision strengths in the threshold region however do not include resonances. Close-coupling techniques, such as the R-matrix method [20], are necessary to provide high precision in this region which is not within the scope of this paper.

With the above qualification, the main limitation of using PWB calculations is that electron exchange between bound and free electrons is not included. The picture of emission is that electron collisions drive excitations within a spin system primarily by dipole transitions, but the balance between the different spin systems is being controlled by the non-allowed transitions between the spin systems. At high-Z, dipole allowed transitions occur between spin systems because of spin system breakdown due to relativistic terms in the Hamiltonian of the target ion (not exchange with the free electron). Mixing occurs between terms of different spin but with equal parity and total angular momentum. However, the high energy behaviour of collision strengths due to true spin-change (i.e. exchange) and those due to spin-system breakdown are not the same. Dipole transitions increase logarithmically with energy while exchange transitions decrease as $1 / E^{2}[21]$. Excluding spin-change transitions will therefore produce the largest discrepancies for the weakly ionised lighter elements.

Only specific spin-change transitions will affect the magnitude of the $\mathcal{P} \mathcal{L} \mathcal{T}$. If the collision strength between a metastable level and a level of opposite spin with a dipole route to the ground level is increased, then population of the metastable will decrease (increasing the $\mathcal{P} \mathcal{L} \mathcal{T}$ ). Increasing the direct collision strength between a metastable and ground level will only setup a local thermodynamic equilibrium (driven by collisional excitation/de-excitation) between the two levels and will not change the population of the metastable.

A comparison between the $\mathcal{P} \mathcal{L} \mathcal{T}^{i c(c s)}$ coefficients calculated using PWB with and without the inclusion of exchange collision strengths (supplemented from AS) is made in figure 7 for three Ar-like ions: $\mathrm{Kr}^{18+}, \mathrm{Xe}^{36+}$ and $\mathrm{W}^{56+}$. The more highly charged ions, $\mathrm{Xe}^{36+}$ and $\mathrm{W}^{56+}$, demonstrate where collision strengths due to spin-system breakdown are comparable to those due to exchange. For the $\mathrm{Kr}^{18+}$ ion, the inclusion of exchange transitions can increase the $\mathcal{P} \mathcal{L} \mathcal{T}$ coefficient by a factor of two and must be included. At higher energies, the two cases converge together for each ion due to the high energy behaviour of the exchange collision strengths.

Ion-impact excitation collision strengths should also be considered when the transition energy is relatively low. Typically, this relates to transitions between finestructure levels and between the $l$ states of high- $n$ shells. For the $\mathcal{P} \mathcal{L} \mathcal{T}$, it is the former that matters when non-statistical fine-structure populations occur. However, as the charge increases within an iso-electronic sequence the transition energy between the fine-structure levels increase and electron-impact excitation effectively mixes the finestructure populations. Further work is planned within ADAS to include ion-impact 


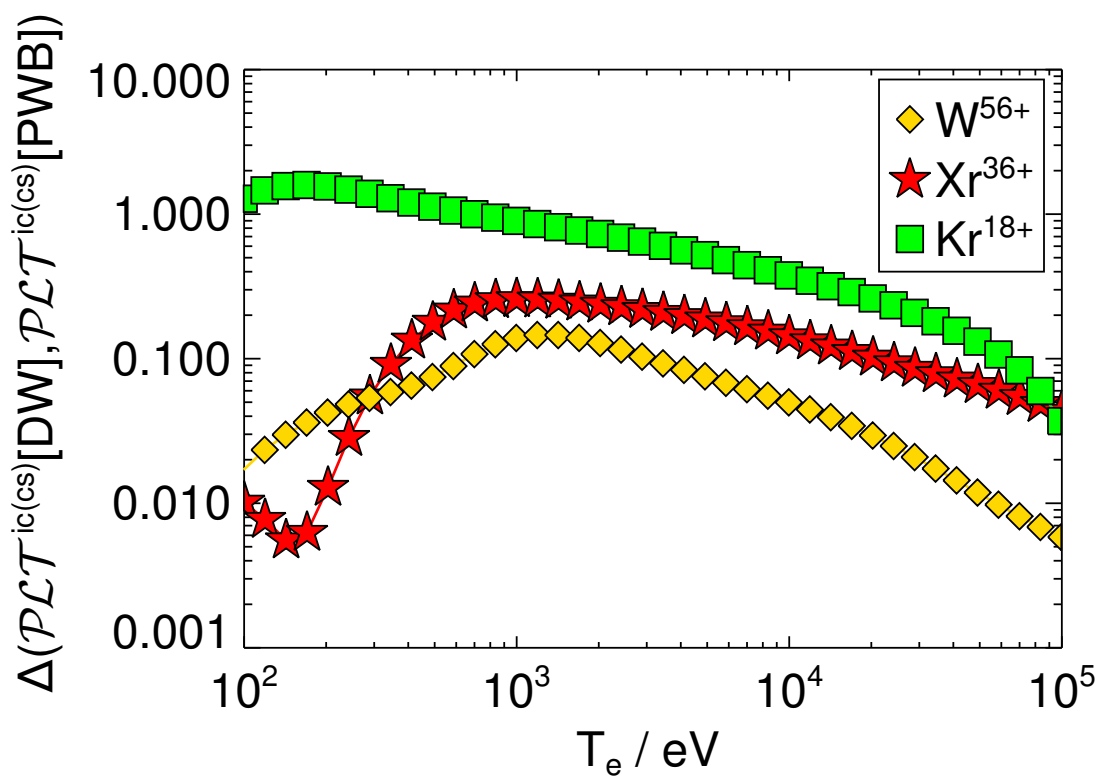

Figure 7. Comparison of the Ar-like $\mathcal{P} \mathcal{L} \mathcal{T}^{i c(c s)}$ coefficients at $n_{e}=10^{19} \mathrm{~m}^{-3}$ generated with and without spin-changing transitions.

excitation collision strengths in the population calculation, however they have not been considered in this analysis. An error of similar magnitude to that found when comparing calculations with and without exchange collision strengths is expected.

\subsection{Atomic structure error}

Although the AS code can produce DW and exchange collision strengths, it is important to assess the quality of its atomic structure and how this impacts on the $\mathcal{P} \mathcal{L} \mathcal{T}$ coefficients. AS is a semi-relativistic code [15,16], whereas CW uses the Hartree-Fock method with relativistic corrections to calculate the Hamiltonian. Using AS in its default state (i.e. without any optimisation) can produce unsatisfactory differences in energy levels in comparison to NIST, generally caused by unrealistic configuration centroid energies. On a case by case basis, it is possible to improve the accuracy of AS, especially for the weakly ionized atoms [22]. That is, AS includes a dimensionless radial scaling parameter for each $n l$-orbital, which can be used to minimise a user specified weighted sum of eigenenergies. This optimisation procedure is not within the scope of this work, but is planned for a future paper.

A similar optimisation can also be applied with $\mathrm{CW}$, where various radial parameters are adjusted to minimise the difference between the Hamiltonian eigenvalues and the experimental energy levels. In this analysis, a set scaling parameters are implemented in $\mathrm{CW}$ providing reasonable solutions (but not exact) for a wide range of ions\|. A comparison between the Ar-like $\mathrm{Fe}^{8+} \mathcal{P} \mathcal{L} \mathcal{T}^{i c(c s)}$ coefficients calculated by $\mathrm{AS}$ and $\mathrm{CW}$ is made in figure 8. The number of transitions used in the population

\|| The scaling parameters used by cowan are defined in adas801 and described in detail by Cowan [13]. 


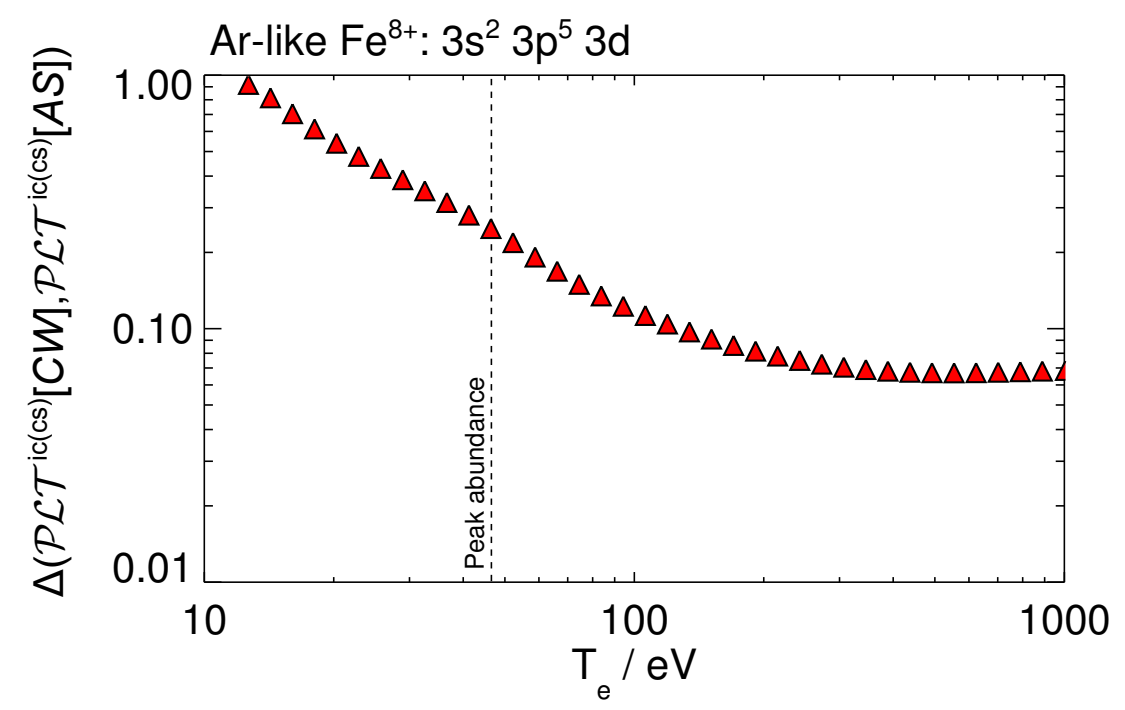

Figure 8. Comparison of the $\mathcal{P} \mathcal{L} \mathcal{T}^{i c(c s)}$ coefficients calculated using $\mathrm{CW}$ and $\mathrm{AS}$ for the Ar-like $\mathrm{Fe}^{8+}$.

calculation with AS data has been reduced to match $\mathrm{CW}$ for a true comparison. At the temperature of peak ion abundance, the $\mathcal{P} \mathcal{L} \mathcal{T}^{i c(c s)}$ from both codes differ by $\approx 20-30 \%$. It is therefore expected that the error from the CW structure (in comparison to the true value) is also $\lesssim 30 \%$.

\section{Data archiving}

The calculations described in this paper are carried out within the ADAS framework and the data is made available via OPEN-ADAS at http://open.adas.ac.uk. Source and output data are stored in ADAS data format ( $a d f$ ) files for which the formal format specification is documented on the ADAS website [9]. The fundamental source data for the atomic energy levels, spontaneous emission coefficients, and effective collision strengths are archived in adf04 files, which are separate files for each ionisation stage in $i c(c s), c a(c s)$, and $c a(c l)$ resolutions. The derived $\mathcal{P} \mathcal{L} \mathcal{T}^{\text {tot }}$ coefficients are archived in iso-nuclear sets with one file per element in the adf11 format.

In ADAS nomenclature, the files will be labelled with the 'year' 41 identifier and author initials: the pattern for adf04 and adf11 will be ssh41_<res $>\#<$ sym $><Z_{1}>$.dat and plt41_<sym $>$.dat, respectively, where $<$ res $>$ is $i c, c a$, or $c l,<$ sym $>$ is ar, fe, $\mathrm{kr}$, xe, or $\mathrm{w}$, and $\left\langle Z_{1}\right\rangle$ is the ion charge from 0 to $Z_{0}-1$.

\section{Conclusions}

A new computerised technique for selecting the configurations which underpin the calculation of the total radiated line power has been presented. The algorithm selects the most important configurations for each ion based on their contribution to the radiated power. Configurations driven by and including the metastables are included and are 
shown to have a varied effect on the radiated power.

The line power coefficients, calculated using the cowan code in intermediatecoupling resolution with (truncated) optimised configuration sets (with a top-up from level bundled calculations using the full configuration sets) have been shown for the isonuclear sequence of a selection of elements: Ar, Fe, Kr, Xe, and W. These coefficients are calculated at one (fusion relevant) electron density $\left(10^{19} \mathrm{~m}^{-3}\right)$ and are typically $20-30 \%$ larger than current predictions for $\mathrm{W}$ from Pütterich and for Kr from Fournier. The practical density range for fusion is no more than two orders of magnitude over which the line power coefficients do not vary significantly. At much higher densities, the population of the metastables should decrease, however this effect can only be modelled with a complete set of excitation data including spin-change, ion-impact, and transitions of higher multipole order.

Calculations using configuration average resolution are shown to be accurate to $\lesssim 10 \%$ except for Pd-like, Kr-like, and Ar-like ions where non-statistical fine-structure populations can significantly lower the radiated power. Plane-wave Born and distorted wave calculations of the radiated power are compared and show that, for near neutral closed-shell ions, the calculations differ substantially (by a factor of two) primarily due to the inclusion of spin-changing (exchange) collision strengths in the distorted wave calculation. Despite the ability of autostructure to calculate more accurate distorted wave collision strengths, the code has not been considered in this analysis for mass production of radiated line power coefficients due to the lack of precision found when running with default parameters $(20-30 \%)$. However, the total radiated line power coefficients for the Pd-like, Kr-like, and Ar-like ions presented in this analysis have been supplemented with spin-changing collision strengths from autostructure.

Finally, this paper has determined the error in the cooling factor expected when using baseline radiated line power coefficients for ions of medium to heavy weight elements. Future work will address the accuracy of the ionisation and recombination coefficients which are used in combination with the line power coefficients to determine the cooling factor.

\section{Acknowledgements}

This work was funded by ADAS and its consortium members.

\section{References}

[1] M. Kovari et al., Fusion Eng. Design. 89, 3054 (2014).

[2] M. Kovari et al., Fusion Eng. Design. 104, 9 (2016).

[3] C. Reux et al., Nucl. Fusion 55, 073011 (2015).

[4] R. A. Pitts et al., Phys. Scr. 2009, 014001 (2009).

[5] R. A. Pitts et al., J. Nucl. Mater. 415, S957 (2012).

[6] G. Federici et al., Fusion Eng. Design. 89, 882 (2014).

[7] A. Kallenbach et al., Plasma Phys. Control. Fusion 55, 124041 (2013).

[8] H. Lux, R. Kemp, D. Ward, and M. Sertoli, Fusion Eng. Design. 101, 42 (2015). 
[9] H. P. Summers, The ADAS manual, version 2.3, http://www.adas.ac.uk, 2001.

[10] H. P. Summers et al., Plasma Phys. Control. Fusion 48, 263 (2006).

[11] T. Pütterich et al., Plasma Phys. Control. Fusion 50, 085016 (2008).

[12] T. Pütterich et al., Nucl. Fusion 50, 025012 (2010).

[13] R. D. Cowan, The Theory of Atomic Structure and Spectra (University of California Press, Berkeley, 1981).

[14] A. R. Foster, Ph.D. thesis, University of Strathclyde, 2008.

[15] N. R. Badnell, J. Phys. B 30, 1 (1997).

[16] N. R. Badnell, Comput. Phys. Commun. 182, 1528 (2011).

[17] R. Radkte et al., Phys. Rev. E 61, 1966 (2000).

[18] K. B. Fournier et al., Nucl. Fusion 40, 847 (2000).

[19] M. M. Bluteau, M. G. O'Mullane, and N. R. Badnell, J. Phys. B 48, 18 (2015).

[20] P. G. Burke, I. Mackey, and I. Shimamura, J. Phys. B: At. Mol. Phys. 10, 2497 (1977).

[21] A. Burgess and J. A. Tully, Astron. Astrophys. 254, 436 (1992).

[22] D. Kasen, N. R. Badnell, and J. Barnes, Astrophys. J. 774, 13 (2013). 